\title{
An online observational study assessing clinical characteristics and impacts of the COVID-19 pandemic on mental health: a perspective study from Bangladesh
}

\author{
Fahmida Begum Mina ${ }^{1} \cdot$ Mutasim Billah ${ }^{1,2}$ (D) Sumon Karmakar ${ }^{3} \cdot$ Sabuj Das ${ }^{1} \cdot$ Md. Siddikur Rahman $^{1}$. \\ Md. Faruk Hasan ${ }^{1,2} \cdot$ Uzzal Kumar Acharjee $^{2}$
}

Received: 12 June 2020 / Accepted: 23 November 2020 / Published online: 7 January 2021

(C) Springer-Verlag GmbH Germany, part of Springer Nature 2021

\begin{abstract}
Purpose The pandemic of coronavirus disease 2019 (COVID-19) has cost numerous lives and induced tremendous mental stress among people. The purpose of this research was to determine anxiety and depression levels, clinical features, and the connections between demographic variables and depression prevalence as well as anxiety prevalence among reported COVID-19 cases in Bangladesh.

Methods For the purpose of data collection, an online cross-sectional survey was carried out from May 26 to June 27, 2020, utilizing a Google adapted preformed questionnaire. The form was shared with a short overview and justification through Facebook, Twitter, Facebook messenger, Viber, and What's App. The Google form contains five parts: a brief introduction, an approval statement, demographics, clinical and radiological data, and mental health assessment by the Generalized Anxiety Disorder 7-item (GAD-7) scale and Patient Health Questionnaire (PHQ-9). Formal ethical clearance was taken from the Institute of Biological Science (IBSc), Bangladesh. Informed consent was ensured before participation.

Results One hundred and fifty-three (153) patients with COVID-19 who had an average age of $39.43 \pm 17.59$ years with male predominance $(72 \%)$ were included. A total of $32.7 \%$ were doing health-care related jobs, and $17.7 \%$ lost their jobs due to COVID-19. Patients had a median income of 30,000 Bangladesh taka (BDT). Of all, 12.4\% of the participants showed asymptomatic features, whereas $87.6 \%$ of patients were symptomatic and presented with fever (79\%), cough (58.8\%), myalgia (24.2\%), breathlessness $(23.5 \%)$, sore throat $(21.6 \%)$, fatigue $(19.6 \%)$, headache $(13.7 \%)$, nausea and/or vomiting $(11.8 \%)$, runny nose $(9.8 \%)$, chest pain $(9.2 \%)$, diarrhea $(8.5 \%)$, stuffy nose (3.2\%), ARDS (2.6\%), oral ulcer (2.6\%), and conjunctivitis (1.9\%). Overall, the prevalence of anxiety and depression was $63.5 \%$ and $56.6 \%$, respectively. Among the participants, $13.2 \%$ had only anxiety, $6.3 \%$ had only depression, and $50.3 \%$ had both.

Conclusion In most cases, middle age, male, and healthy workers were patients. Fever and cough were the standard presentations. Approximately two-thirds or $66.67 \%$ of patients had anxiety and depression, one or both.
\end{abstract}

Fahmida Begum Mina and Mutasim Billah contributed equally to this work.

Mutasim Billah

mutasimbillahshazu@gmail.com

Md. Faruk Hasan

faruk_geb@yahoo.com

1 Department of Genetic Engineering and Biotechnology, University of Rajshahi, Rajshahi 6205, Bangladesh

2 Joarder DNA \& Chromosome Research Laboratory, University of Rajshahi, Rajshahi 6205, Bangladesh

3 Molecular Biology and Protein Science Laboratory, University of Rajshahi, Rajshahi 6205, Bangladesh
Keywords COVID-19 · Bangladesh · Clinical characteristics . Anxiety $\cdot$ Depression

\section{Background}

A number of pneumonia cases of suspected viral origin were found in Wuhan city, Hubei, China by December 2019 (Huang et al. 2020). This disease was identified first as novel coronavirus (2019-nCoV disease) which was later renamed as the coronavirus disease 2019 (COVID-19) under a global agreement and the virus was identified as SARS-CoV-2 (Siordia 2020). Bangladesh declared the first COVID-19 affected case three days earlier than the WHO's global announcement of the COVID-19 Pandemic on 11 March 2020 
(Mina et al. 2020). More than 19,849,259 cases and 730,420 deaths had been documented worldwide by August 09, 2020, and 3399 deaths were reported in Bangladesh out of 257,600 confirmed cases (WHO 2020a, b). COVID-19 pandemic trajectory, the predicted number of cases and deaths or to what extent quarantine measures will clash with daily life remain uncertain for the experts. The clinical appearance of COVID19 induced infection ranges from asymptomatic disease to extreme respiratory trouble and shock (Huang et al. 2020; Banna et al. 2020; Chen et al. 2020; Chan et al. 2020). Symptomatic cases are associated initially with fever, respiratory symptoms, e.g., Breathe shortness and cough, and are linked to different proportions of dyspnea, headache, myalgia, rhinorrhea, sore throat, nausea, and/or vomiting (Chen et al. 2020; Chan et al. 2020; Zhu et al. 2020a, b; RodriguezMorales et al. 2020). Furthermore, patients also show neurological symptoms, such as ataxia, convulsion (Baig 2020), and some other neurological issues, such as ischaemic or haemorrhagic stroke, dizziness, impaired mental condition, Guillain-Barré syndrome, or acute necrotising encephalopathy (Mao et al. 2020; Poyiadji et al. 2020; Li et al. 2020). Within $2-40 \%$ of the patients, gastrointestinal symptoms are reported in case series (Guan et al. 2020; Zhang et al. 2020) and diarrhea may be the primary presentation of infection (Song et al. 2020). In a number of centers, tasting or olfactory disorders were also reported (Giacomelli et al. 2020). During the outbreaks, cardiovascular symptoms, for example, arrythmia, decreased cardiac activity, myocardial inflammation or injury have also been recorded (Inciardi et al. 2020). A new retrospective observational analysis in China showed that COVID19 positive patients had a hypercoagulable condition (Zhou et al. 2020; Vetter et al. 2020). A large number of Bangladeshi citizens are especially vulnerable to SARS-CoV-2 because of their high population density, improper personal hygienic habits, and poor financial conditions. Fear of sickness, the lockdown isolation, the economic need to work, and the failure to ensure public access for necessary things such as food can contribute to increased psychological issues for the general population. In addition to physical manifestations, a fair number of patients experience psychiatric disorders and/or complications, particularly elderly people and persons with low incomes are at greater risk for impaired mental health (Zhu et al. 2020a, b; Wang et al. 2020; Kong et al. 2020). The available published psychological effect research for COVID-19 centered particularly on healthcare professionals and the general population but not on confirmed COVID-19 patients (Zhu et al. 2020a, b; Wang et al. 2020). The latest studies from China show that $31.9 \%$ and $33.7 \%$ of COVID19-positive patients have anxiety and depression symptoms (Salari et al. 2020). Symptoms severity are also related to different sociodemographic variables, including gender, age, and social assistance (Kong et al. 2020). There is a clear difference in sociodemographic features between countries
(Huang et al. 2020; Siordia 2020; Banna et al. 2020; Baig 2020; Mao et al. 2020), and the management approach of one country may not be applicable in another. Therefore, it is important to know the specific features of each country for the strategic planning and proper execution. Although several published reports included different perspectives of COVID19 patients from various countries, none from Bangladesh has yet been reported.

In this current study, we aimed to assess COVID-19 clinical manifestations, anxiety, and depression percentages and the association between demographic factors, depression, and anxiety in reported COVID-19 cases from Bangladesh. This scientific study may help scientists and policy makers to gather an overview of the country's scenarios and may help to raise consciousness about this pandemic.

\section{Materials and methods}

The Institute of Epidemiology, Diseases Control and Research (IEDCR) announced the first COVID 19-positive case in Bangladesh on 8 March 2020. The number of COVID-19 cases rose at an unprecedented pace at the end of March. Consequently, since 26 March, the Government of the People's Republic of Bangladesh has declared general holidays/lockdown, which at that time was modeled on the basis of the situation (TSB 2020). Regardless of the lockdown, data collection from health clinics or regional screening samples from neighborhood facilities is not achievable. A crosssectional electronic survey method was therefore adopted from May 26 to June 27, 2020. COVID-19 positive people, aged $>18$ years old who were using Facebook (the most commonly accessed social media in the country) were primarily chosen as the target category. Moreover, people using Twitter, Viber, WhatsApp, and other online communication portals were also approached. The author's "Facebook" news feed shared a recruiting message. It was then posted by the entire author team on "Twitter" and other parties via their contacts in "Facebook Messenger," "WhatsApp," and "Viber." This post included a quick overview of the context, purpose, processes, volunteer essence of involvement, confidentiality and secrecy claims, questionnaire completion notes, and the link to the online questionnaire (Google Form). The contents of the letter were understood and accepted by the participants in the study, who are citizens of Bangladeshi nationality, instructed to complete a survey by clicking on the link. Moreover, via short message services (SMS), the questionnaire link or the link leading to the Google Form was circulated to as many of the respondents as possible. The Google form consists of five parts: (i) a brief introduction, (ii) consent statement, (iii) sociodemographic characteristics of the participants, which include: age, sex, residence, education, occupation, monthly income, number of family members, suspected place of 
contact, and travel history of the participants, (iv) clinical features, which include: asymptomatic and symptomatic features, various types of symptoms, such as fever, cough, breathlessness, myalgia, sore throat, fatigue, nausea and/or vomiting, headache, runny nose, chest pain, diarrhea, ARDS, stuffy nose, conjunctivitis, and oral ulcer; also radiological information, such as, chest X-ray, CT scan of the chest, and other existing co-morbidities of the participants, (v) mental health assessment by the Generalized Anxiety Disorder 7-item (GAD-7) scale (Johnson et al. 2019) and Patient Health Questionnaire (PHQ-9) (Levis et al. 2019). The GAD-7 is a valid and efficient tool for screening for GAD and assessing its severity in clinical practice and research. The GAD-7 scale is a valid and reliable tool for screening of anxiety and assessing its severity in epidemiological surveys. The 7 items scale of the Bangla Generalized Anxiety Disorder assessment questionnaire (Haque et al. 2014) was used to assess the level of anxiety. This scale consists of 7 items answered on a fourpoint Likert scale ranging from 0 ("not at all") to 3 ("nearly every day"). The level of anxiety was categorized into four groups as minimal, mild, moderate, and severe based on scoring $0-4,5-9,10-14$, and $15-21$, respectively. In this study, those scoring $(\geq 10)$ moderate to extremely severe were placed to determine the existence of anxiety, similar to levels of depression on the PHQ-9.

For the evaluation of the frequency and extent of anxiety and depression among the participants, the GAD-7 and PHQ-9 questionnaires were used. In order to notify the post regarding any difficulties to completing the evaluation form, a direct email address and a contact number have been given in the post. Initially, ten random participants completed a questionnaire pretest, using the pilot experience to make additional changes before the final online release of Facebook's post. The form has been set in order not to comply with the following sections of the questionnaire when anyone clicks the link to join and does not agree with the informed consent declarations. Hence, a total of 437 responses were found, and only 153 respondents actually agreed and completed the form. These 153 responses were finally included in the study. Eight data points were filled by the relatives of the deceased persons.

Ethics statement Before the commencement of the study, formal ethical approval was obtained from the Ethical Review Committee (ERC) of the Institute of Biological Science (IBSc), Bangladesh. In accordance with the new Helsinki Declaration, all approaches were introduced. Prior to registration, all respondents gave written permission.

Data cleaning and analyses Because the Google process is automatic, data fill up is processed as "comma separate value" (csv) format in Google drive as sheets. The sheet was cleaned, assembled, and imported into the statistical software for social science (SPSS) 25 (SPSS Inc., Chicago, IL, USA) for final analysis. Descriptive statistics were used during analysis, where continuous variables were expressed as the mean \pm standard deviation and categorical variables were expressed as count (percentage).

\section{Results}

A total of 153 persons infected with COVID-19 were included in this study. The mean age of the patients was $39.43 \pm$ 17.59 years. The majority of patients were aged $<30$ years $(28.1 \%)$. Among all, $72 \%$ of the patients were male, and $56.2 \%$ came from the urban areas and $19.6 \%$ were from rural areas. Nearly, $13.7 \%$ patients received no formal schooling and $30 \%$ of patients completed graduation and above. The majority of patients were doing private service $(29.4 \%)$ and $9.8 \%$ of the participants were unemployed. Among the participants, $32.7 \%$ had healthcare-related jobs, and $17.7 \%$ lost their jobs due to the presence of COVID-19. The median monthly family income of patients was 30,000 BDT, with the majority having incomes less than 25,000 BDT (39.9\%). Nearly half of the participants had a family of four or more people (49.1\%). The most common suspected place of contact was the workplace $(47.1 \%), 18.9 \%$ was from market places, $12.5 \%$ of the suspected place of contact was unknown, and $1.2 \%$ had a travel history (Table 1).

Table 2 lists the preventive measures taken by patients for COVID-19. Proper social distancing practice, putting up of masks, hand-washing, refrain from touching eyes, nose or mouth and covering nose and mouth with bent elbow or tissue while coughing or sneezing was practiced by $86.3,83.7,82.4$, 83 , and $81 \%$ of the respondents, respectively.

COVID-19 symptoms were present in $87.6 \%$ of the participants. The most common presenting symptom was fever (79\%), followed in decreasing order by cough (58.8\%), myalgia $(24.2 \%)$, breathlessness $(23.5 \%)$, sore throat $(21.6 \%)$, fatigue (19.6\%), headache (13.7\%), nausea and/or vomiting $(11.8 \%)$, runny nose $(9.8 \%)$, chest pain $(9.2 \%)$, diarrhea (8.5\%), stuffy nose (3.2\%), ARDS (2.6\%), oral ulcer (2.6\%), and conjunctivitis $(1.9 \%)$. Chest X-ray was performed in $25.4 \%$ of patients, among who 46.2, 35.9, and $17.9 \%$ had normal chest X-ray, bilateral pneumonia, and unilateral pneumonia, respectively. A CT scan of the chest was performed in five patients, and all had bilateral pneumonia. Among all, $41.8 \%$ had co-morbidities, among who 22.9, 14.4, 11.1, and $0.6 \%$ had endocrine, respiratory, cardiovascular, and hematological disease (Table 3).

Patients were most commonly treated with an antibiotic (64\%), followed in second by hydroxychloroquine (27.5\%). Antiviral and corticosteroids were given to 5.9 and $2.6 \%$ patients, respectively. Oxygenation was needed in $7.8 \%$ of patients. Overall, $18.9 \%$ of patients had an outcome at the time of data collection. Among those, $72.4 \%$ were discharged 
Table 1 Sociodemographic characteristics of individuals with COVID-19 $(n=153)$

\begin{tabular}{|c|c|}
\hline Variables & Findings \\
\hline Age (years) & $39.43 \pm 17.59$ \\
\hline \multicolumn{2}{|l|}{ Age groups } \\
\hline$<30$ & $43(28.1)$ \\
\hline $31-40$ & $37(24.2)$ \\
\hline $41-50$ & $27(17.7)$ \\
\hline $51-60$ & $23(15.0)$ \\
\hline$>60$ & $23(15.0)$ \\
\hline \multicolumn{2}{|l|}{ Sex } \\
\hline Male & $110(72)$ \\
\hline Female & $43(28)$ \\
\hline \multicolumn{2}{|l|}{ Residence } \\
\hline Urban & $86(56.2)$ \\
\hline Semi urban & $87(24.2)$ \\
\hline Rural & $30(19.6)$ \\
\hline \multicolumn{2}{|l|}{ Education } \\
\hline No formal schooling & $21(13.7)$ \\
\hline Primary & $31(20.4)$ \\
\hline SSC or similar & $38(24.8)$ \\
\hline HSC or similar & $17(11.1)$ \\
\hline Graduation & $34(22.2)$ \\
\hline Post-graduation & $12(7.8)$ \\
\hline \multicolumn{2}{|l|}{ Occupation } \\
\hline Private service & $45(29.4)$ \\
\hline Government service & $35(22.9)$ \\
\hline Businessman & $31(20.3)$ \\
\hline Housewife & $21(13.7)$ \\
\hline Student & $6(3.9)$ \\
\hline Unemployed & $15(9.8)$ \\
\hline Health-care related job & $50(32.7)$ \\
\hline Became unemployed due to COVID-19 & $17(17.7)$ \\
\hline Monthly income (BDT) & $30,000(16000-50,000)$ \\
\hline$<25,000$ & $55(39.9)$ \\
\hline 25,000 to 50,000 & $53(38.4)$ \\
\hline 50,001 to 75,000 & $15(10.9)$ \\
\hline$>75,000$ & $15(10.9)$ \\
\hline \multicolumn{2}{|c|}{ Number of family members living in the same house } \\
\hline One & $12(7.8)$ \\
\hline Two to four & $66(43.1)$ \\
\hline$\geq$ Four & $75(49.1)$ \\
\hline \multicolumn{2}{|l|}{ Suspected place of contact } \\
\hline Workplace & $72(47.1)$ \\
\hline Market place/bazar & $29(18.9)$ \\
\hline Home & $15(9.8)$ \\
\hline Hospital & $12(7.8)$ \\
\hline Mosque & $6(3.9)$ \\
\hline Unknown & $19(12.5)$ \\
\hline Travel history (outside country) & $25(1.2)$ \\
\hline
\end{tabular}

Continuous data is expressed as mean $\pm \mathrm{SD}$ or median (IQR) and frequency data is expressed as counts (percentage) 
Table 2 Preventive measures practices of individuals with COVID-19 $(n=153)$

\begin{tabular}{lc}
\hline Variables & Findings \\
\hline Social distancing practice & $132(86.3)$ \\
Always & $17(11.1)$ \\
Not always & $4(2.6)$ \\
No & \\
Putting up mask & $128(83.7)$ \\
Always & $17(11.1)$ \\
Not always & $8(5.2)$ \\
No & \\
Washing hands & $126(82.4)$ \\
Frequent & $21(13.7)$ \\
Not frequent & $6(3.9)$ \\
No & \\
Refrain from touching eyes, nose or mouth & $127(83)$ \\
Frequent & $19(12.4)$ \\
Not frequent & $7(4.6)$ \\
No & $124(81)$ \\
Covering nose and mouth with bent elbow or tissue while coughing or sneezing & $21(13.7)$ \\
Frequent & $8(5.3)$ \\
Not frequent & \\
No &
\end{tabular}

Data is expressed as counts (percentage) without complications, and $27.6 \%$ died. Among those who were being treated, $45.2 \%$ were isolated at home, $39.5 \%$ were being treated in dedicated COVID-19 hospitals, and $15.3 \%$ were being managed at general hospitals (Table 4).

Anxiety and depression were assessed among 145 living patients. The prevalence of anxiety and depression was 63.5 and $56.6 \%$, respectively. Among all, 50.3\% had both anxiety and depression, $13.2 \%$ had only anxiety, and $6.3 \%$ had only depression. Among patients with anxiety, 58.7\%, 18.5\%, and $22.8 \%$ had mild, moderate, and severe anxiety, respectively. Among patients with depression, 48.8, 37.8, 4.9, and 8.5\% had mild, moderate, moderately severe, and severe depression, respectively (Table 5).

\section{Discussion}

We extracted data from a total of 153 Bangladeshi patients with COVID-19 confirmed through RT-PCR from May 26 to June 27, 2020. The average age of all patients was $39.43 \pm$ 17.59 years, with a majority belonging to $<30$ and $31-$ 40 years $(52.3 \%)$. The Institute of Epidemiology, Disease Control, and Research (IEDCR) data revealed that $66.5 \%$ of the Bangladeshi COVID-19 cases were aged between 21 and 50 years (IEDCR 2020). This COVID-19 age distribution in Bangladesh is comparable to that in India, but varies from that in the United States, China and Spain, among the countries that are severely affected with SARS-CoV-2 virus (Maswood 2020). This may be because the younger group in Bangladesh ignored government orders and prohibition, wrongly deemed exempt from COVID-19, and the lower middle age (27.1 years) as opposed to other countries, could be another factor (Index Mundi 2020). We observed a male predominance (72\%) consistent with the COVID-19 status of Bangladesh (male 69\%) (IEDCR 2020), indicating a strong gender discrepancy in COVID-19 case identification of the adult population in Bangladesh. It can be exacerbated by Bangladesh's social history, where a man is in most situations the only wage earner in his family and wants more autonomy compared with a woman. An analytical report by Hossain et al. regarding COVID-19 cases in Bangladesh indicated that the underreporting of female patients due to timidness and/or social stigma even after infections is an issue that leads to rapid progression of the disease (Hossain et al. 2020). However, studies from other countries also found a greater number of male patients with COVID-19 infection than female (Chen et al. 2020; Garg et al. 2020; Badawi and Ryoo 2016; Channappanavar et al. 2017) MERSCoV and SARS-CoV had a similar pattern of sex distribution (Badawi and Ryoo 2016; Channappanavar et al. 2017) as different factors have been shown to account for the sex-based disparity in immune responses, including genetic factors and hormonal mediators (Jaillon et al. 2019). 
Table 3 Clinical features of patients with COVID$19(n=153)$

\begin{tabular}{|c|c|}
\hline Variables & Findings \\
\hline \multicolumn{2}{|l|}{ Presentation } \\
\hline Asymptomatic & $19(12.4)$ \\
\hline Symptomatic & $134(87.6)$ \\
\hline \multicolumn{2}{|l|}{ Symptoms } \\
\hline Fever & $121(79)$ \\
\hline Cough & $90(58.8)$ \\
\hline Myalgia & $37(24.2)$ \\
\hline Breathlessness & $36(23.5)$ \\
\hline Sore throat & $33(21.6)$ \\
\hline Fatigue & $30(19.6)$ \\
\hline Headache & 21(13.7) \\
\hline Nausea and/or vomiting & 18(11.8) \\
\hline Runny nose & $15(9.8)$ \\
\hline Chest pain & $14(9.2)$ \\
\hline Diarrhea & $13(8.5)$ \\
\hline Stuffy nose & $5(3.2)$ \\
\hline ARDS & $4(2.6)$ \\
\hline Oral ulcer & $4(2.6)$ \\
\hline Conjunctivitis & $3(1.9)$ \\
\hline \multicolumn{2}{|l|}{ Investigation } \\
\hline \multicolumn{2}{|l|}{ Chest X-ray } \\
\hline Done in & $39(25.4)$ \\
\hline Normal & $18(46.2)$ \\
\hline Bilateral pneumonia & 14(35.9) \\
\hline Unilateral pneumonia & $7(17.9)$ \\
\hline \multicolumn{2}{|l|}{ CT scan of chest } \\
\hline Done in & $5(3.3)$ \\
\hline Bilateral pneumonia & $5(100)$ \\
\hline \multicolumn{2}{|l|}{ Co-morbidities } \\
\hline Present & $64(41.8)$ \\
\hline Endocrine disease & $35(22.9)$ \\
\hline Respiratory disease & $22(14.4)$ \\
\hline Cardiovascular disease & $17(11.1)$ \\
\hline Hematological disease & $1(0.6)$ \\
\hline
\end{tabular}

Data is expressed as counts (percentage)

Among the participants, $32.7 \%$ had healthcare-related jobs, with the most common suspected place of contact being the workplace (47.1\%). Hossain et al. found $25.66 \%$ health workers among all COVID-19 cases of Bangladesh, ranking hospital physicians on top followed by nurses (Hossain et al. 2020). This exposure positions healthcare staff at the main infection vector, requiring that appropriate precautions be taken to minimize the chance of infection (Hasan 2020). Therefore, a fast detection of asymptomatic infections should be checked between health workers before and after SARSCOV-2 exposure (Huang et al. 2020).

In this study, $87.6 \%$ of participants were symptomatic, wherein the most commonly reported symptoms were fever,

Table 4 Management and clinical status of persons with COVID-19 $(n=153)$

\begin{tabular}{lr}
\hline Variables & Findings \\
\hline Treatment history & \\
Antibiotic & $98(64)$ \\
Hydroxychloroquine & $42(27.5)$ \\
Oxygen & $12(7.8)$ \\
Antiviral & $9(5.9)$ \\
Corticosteroid & $4(2.6)$ \\
Clinical status & \\
Outcome present & $29(18.9)$ \\
Currently under treatment & $124(81.1)$ \\
Treatment place & \\
Home isolation & $56(45.2)$ \\
Being treated in COVID-19 hospital & $49(39.5)$ \\
Being treated in general hospital & $19(15.3)$ \\
Outcome & \\
Discharged without complication & $21(72.4)$ \\
Died & $8(27.6)$ \\
\hline
\end{tabular}

Data is expressed as counts (percentage)

cough, breathlessness, myalgia, sore throat, and fatigue. Less commonly reported symptoms included headache, nausea and/or vomiting, chest pain, runny nose, diarrhea, stuffy nose, ARDS, oral ulcer, and conjunctivitis. Several studies also

Table 5 Anxiety and depression among persons with COVID-19 $(n=$ 145)

\begin{tabular}{lc}
\hline Variables & Findings \\
\hline Anxiety disorder & \\
Prevalence & $92(63.5)$ \\
Severity & \\
Mild anxiety (GAD7: 5-9) & $54(58.7)$ \\
Moderate anxiety (GAD7: 10-14) & $17(18.5)$ \\
Severe (GAD7: 15-21) & $21(22.8)$ \\
Depressive disorder & \\
Prevalence & $82(56.6)$ \\
Severity & \\
Mild (PHQ9: 5-9) & $40(48.8)$ \\
Moderate (PHQ9: 10-14) & $31(37.8)$ \\
Moderately severe (15-19) & $4(4.9)$ \\
Severe (20-27) & $7(8.5)$ \\
Distributions & \\
Both anxiety and depression & $73(50.3)$ \\
Only anxiety & $19(13.2)$ \\
Only depression & $9(6.3)$ \\
None & $44(30.3)$ \\
\hline
\end{tabular}

Data is expressed as counts (percentage) 
found similar clinical presentations, mostly fever, dry cough, and dyspnea (Vetter et al. 2020; Garg et al. 2020; Singhal 2020), correlating clinical features between COVID-19 and previous beta coronavirus infections (Huang et al. 2020). Nonetheless, the entire spectrum of clinical effects is not known yet, as the signs recorded indicate a vast variety of moderate to severe and in many cases asymptomatic signs (Mizumoto et al. 2020; Ng et al. 2020; Lippi et al. 2020; Nishiura et al. 2020; Zou et al. 2020). We found that $12.4 \%$ of our study patients were asymptomatic, which is comparatively lower than that found in earlier cited studies (Mizumoto et al. 2020; Oran and Topol 2020; Long et al. 2020;Nishiura et al. 2020). In Bangladesh, it is permissible to check COVID19 for only symptomatic patients and people with experience of interaction with reported cases, owing in particular to the lack of RT-PCR kits and laboratory facilities (Mina et al. 2020). For treating SARS-CoV-2 contaminated cases, no drug regime has been authorized yet (Singhal 2020; Kolifarhood et al. 2020), although our national guideline for clinical management of COVID-19 promoted supportive and symptomatic treatment protocols along with judicial use of different modalities of drug regimen found to be effective by different trials (DGHS 2020). This sequence has been accompanied by more than half of patients getting hydroxychloroquine with antibiotics; also, antiviral and corticosteroids were given in a fewer number of cases. Oxygenation was required in $7.8 \%$ of cases.

We found a case-fatality rate of $5.23 \%$ out of 153 patients, though the rate is higher $(28.6 \%)$ according to methods for estimating the case-fatality ratio for a novel, emerging infectious disease (Ghani et al. 2005). Nevertheless, the case fatality rate is frequently underestimated during the early stages of the pandemic because the identification is primarily geared toward the more severe cases (Worldometer reports 2020). Proper social distancing practice, putting up of mask, handwashing, refrain from touching eyes, nose or mouth, and covering nose and mouth with bent elbow or tissue while coughing or sneezing was practiced by $86.3,83.7,82.4,83$, and $81 \%$ of the respondents, respectively. This high visibility is largely attributed to the fact that government and media have been able to access knowledge about the virus since the epidemic began, as well as to its contraction. Another explanation could be that $65.9 \%$ of students received a degree of academic study backed by the positive correlation between educational attainment and COVID-19 awareness (Zhong et al. 2020; Erfani et al. 2020). Among the study participants, 63.5 and $56.6 \%$ had anxiety and depression, respectively. Among those with anxiety, 58.7, 18.5, and $22.8 \%$ had mild, moderate, and severe anxiety, respectively, and among those with depression, 48.8, 37.8, 4.9, and $8.5 \%$ had mild, moderate, moderately severe, and severe depression, respectively. A similar report (Liu et al. 2020) was noticed in China, where in cases with a total of $53.8 \%$, respondents suffered from moderate to severe epidemic psychology; $16.5 \%$ reported mild to serious depression symptoms; $28.8 \%$ reported moderate to severe symptoms of anxiety; and $8.1 \%$ report moderate to severe level of stress. While the major public psychological consequences to date are increased rates of stress and depression, when new interventions and impacts for spread reduction, in particular quarantine, are implemented and influence the habits or living circumstances of many individuals, the levels of isolation, depression, unhealthy intake of alcohol, narcotics, and self-harm or suicide are still anticipated to develop (WHO 2020a, b).

\section{Conclusion}

This series mainly featured medium-aged individuals, males, and medical professionals. In more than $80 \%$ of the patients, preventative procedures were maintained. Fever and cough were the most common symptoms, with a significant proportion of patients with breathing problems, sore throat, and exhaustion. The treatment was frequently carried out with antibiotics and hydroxychloroquine. Among COVID-19 affected patients, depression and anxiety were prominent, as approximately twothirds of patients had one or both.

\section{Limitations}

There are several limitations to this study. First, 153 patients have been included with confirmed cases regarding COVID-19 and medically presumed, but undiagnosed cases have been removed. Second, comprehensive information about patients was not available, particularly on the clinical outcomes, because most patients were hospitalized when we submitted the manuscript. Third, an oversampling of a certain network of peers (for example socially involved) occurred, and it can therefore be treated as a selection bias, which adds another limitation to this research. The conclusion is therefore less widespread to the whole population, especially to less educated individuals. One other limitation is that self-reported degrees of psychological effects, anxiety, depression, and stress are not always consistent with the evaluation by specialists in mental health.

\section{Future scope and recommendation of the study}

The findings of this analysis provide an early evaluation of the epidemiological and clinical features of Bangladesh's COVID-19 cases. Further research studies are required to gain an overview of clinical severity in outpatients, primary care, and community settings.

Acknowledgments The author thanks the patients who participated in the study during this stressful situation. The authors are also thankful to the Institute of Biological Sciences (IBSc) for support throughout the study period. 


\section{Compliance with ethical standards}

Conflict of interests The authors declare they have no conflict of interest.

Ethical consideration Ethical measures were taken throughout the study period to maintain a high standard of confidentiality and anonymity of the participants. Formal ethical clearance was taken from the ethical review committee of the Institute of Biological Science (IBSc) for conducting the study, and formal permission was taken from the responders through Google Form.

\section{References}

Badawi A, Ryoo SG (2016) Prevalence of comorbidities in the Middle East respiratory syndrome coronavirus (MERS-CoV): a systematic review and meta-analysis. Int J Infect Dis 49:129-133. https://doi. org/10.1016/j.ijid.2016.06.015

Baig AM (2020) Neurological manifestations in COVID-19 caused by SARS-CoV-2. CNS Neurosci Ther 26(5):499-501. https://doi.org/ $10.1111 /$ cns. 13372

Banna MHA, Sayeed A, Kundu S et al (2020) The impact of the COVID19 pandemic on the mental health of the adult population in Bangladesh: a nationwide cross-sectional study. Int J Environ Health Res. https://doi.org/10.1080/09603123.2020.1802409

Chan JF et al (2020) A familial cluster of pneumonia associated with the 2019 novel coronavirus indicating person-to-person transmission: a study of a family cluster. Lancet 395(10223):514-523. https://doi. org/10.1016/S0140-6736(20)30154-9

Channappanavar R, Fett C, Mack M et al (2017) Sex-based differences in susceptibility to severe acute respiratory syndrome coronavirus infection. J Immunol 198(10):4046-4053. https://doi.org/10.4049/ jimmunol.1601896

Chen N, Zhou M, Dong X et al (2020) Epidemiological and clinical characteristics of 99 cases of 2019 novel coronavirus pneumonia in Wuhan , China : a descriptive study. Lancet 395(10223):507513. https://doi.org/10.1148/radiol.2020201187

DGHS (2020) National Guidelines on Clinical Management of Coronavirus Disease 2019. https://dghs.gov.bd/index.php/en/ home/5376-novel-coronavirus-covid-19-guidelines

Erfani A, Shahriarirad R, Ranjbar K et al (2020) Knowledge, attitude and practice toward the novel Coronavirus (COVID-19) outbreak: a population-based survey in Iran. https://doi.org/10.2471/BLT.20. 256651

Garg S, Kim L, Whitaker M et al (2020) Hospitalization rates and characteristics of patients hospitalized with laboratory-confirmed coronavirus disease 2019 - COVID-NET, 14 states, march 1-30, 2020. MMWR Morb Mortal Wkly Rep 69(15):458-464. https://doi.org/ 10.15585/mmwr.mm6915e3

Ghani AC, Donnelly CA, Cox DR et al (2005) Methods for estimating the case fatality ratio for a novel, emerging infectious disease. Am J Epidemiol 162(5):479-486. https://doi.org/10.1093/aje/kwi230

Giacomelli A, Pezzati L, Conti F et al (2020) Self-reported olfactory and taste disorders in patients with severe acute respiratory coronavirus 2 infection: a cross-sectional study. Clin Infect Dis 71(15):889-890. https://doi.org/10.1093/cid/ciaa330

Guan WJ, Ni ZY, Hu Y et al (2020) Clinical characteristics of coronavirus disease 2019 in China. N Engl J Med 382(18):1708-1720. https:// doi.org/10.1056/NEJMoa2002032

Haque MJ, Das CK, Ara R et al (2014) Prevalence of generalized anxiety disorder and its effect on daily living in the rural community of Rajshahi. J Teach Assoc 27(1):14-23
Hasan K (2020) BMA Healthcare workers account for $11 \%$ of Covid-19 cases. https://www.dhakatribune.com/health/coronavirus/2020/04/ 27/healthcare-workers-account-for-11-of-covid-19-cases-bma

Hossain A, Rana J, Benzadid S et al (2020) COVID-19 and Bangladesh-Analytical report-3. https://drive.google.com/file/d/ 1o0-Zim0V7m9qb6Ijq tx7Q9scCJIaRw2/view

Huang C, Wang Y, Li X et al (2020) Clinical features of patients infected with 2019 novel coronavirus in Wuhan, China. Lancet 395(10223): 497-506. https://doi.org/10.1016/S0140-6736(20)30183-5

IEDCR (2020) Covid-19 status Bangladesh. https://www.iedcr.gov.bd/

Inciardi RM, Lupi L, Zaccone G et al (2020) Cardiac involvement in a patient with coronavirus disease 2019 (COVID-19). JAMA Cardiol 5(7):1-6. https://doi.org/10.1001/jamacardio.2020.1096

Index Mundi (2020) World Factbook. https://www.indexmundi.com/ factbook

Jaillon S, Berthenet K et al (2019) Sexual dimorphism in innate immunity. Clin Rev Allergy Immunol. 56(3):308-321. https://doi.org/10. 1007/s12016-017-8648-x

Johnson SU, Ulvenes PG, Øktedalen T et al (2019) Psychometric properties of the general anxiety disorder 7-item (GAD-7) scale in a heterogeneous psychiatric sample. Front Psychol. 10:1713. https:// doi.org/10.3389/fpsyg.2019.01713

Kolifarhood G, Aghaali M, Saadati HM et al (2020) A narrative review. Clin Asp COVID. 8(1):41 http://journals.sbmu.ac.ir/aaem

Kong X, Zheng K, Tang M et al (2020) Prevalence and factors associated with depression and anxiety of hospitalized patients with COVID19. MedRxiv. https://doi.org/10.1101/2020.03.24.20043075

Levis B, Benedetti A et al (2019) Collaboration. Accuracy of patient health questionnaire-9 (PHQ-9) for screening to detect major depression: individual participant data meta-analysis. BMJ 365: 11476. https://doi.org/10.1136/bmj.11476

Li Y, Li M, Wang M et al (2020) Acute cerebrovascular disease following COVID-19: a single center, retrospective, observational study. Stroke Vasc Neurol. https://doi.org/10.1136/svn-2020-000431

Lippi G, Sanchis-Gomar F, Henry BM (2020) Coronavirus disease 2019 (COVID-19): the portrait of a perfect storm. Ann Transl Med. 8(7): 497. https://doi.org/10.21037/atm.2020.03.157

Liu X, Luo WT, Li Y et al (2020) Psychological status and behavior changes of the public during the COVID-19 epidemic in China. Infect Dis Poverty 9, 58. https://doi.org/10.1186/s40249-02000678-3

Long QX, Tang XJ, Shi QL et al (2020) Clinical and immunological assessment of asymptomatic SARS-CoV-2 infections. Nat Med. https://doi.org/10.1038/s41591-020-0965-6

Mao L, Jin H, Wang M et al (2020) Neurologic manifestations of hospitalized patients with Coronavirus Disease 2019 in Wuhan, China. JAMA Neurol. 77(6):1-9. https://doi.org/10.1001/jamaneurol.2020. 1127

Maswood MH (2020) Young, working-age people mostly infected with COVID-19 in Bangladesh. NEWAGE/Bangladesh. https://www. newagebd.net/article/104307/young-working-age-people-mostlyinfected-with-covid-19-in-bangladesh

Mina FB, Billah M, Rahman MS et al (2020) COVID-19: transmission, diagnosis, policy intervention, potential broader perspective on the rapidly evolving situation in Bangladesh. J Adv Biotechnol Exp Ther 3(4):18-29. https://doi.org/10.5455/jabet.2020.d152

Mizumoto K, Kagaya K, Zarebski A et al (2020) Estimating the asymptomatic proportion of coronavirus disease 2019 (COVID-19) cases on board the Diamond Princess cruise ship, Yokohama, Japan, 2020. Euro Surveill 25(10):2000180. https://doi.org/10.2807/15607917.ES.2020.25.10.2000180

Ng OT, Marimuthu K, Chia PY et al (2020) SARS-CoV-2 infection among travelers returning from Wuhan, China. N Engl J Med. 382(15):1476-1478. https://doi.org/10.1056/NEJMc2003100

Nishiura H, Kobayashi T, Miyama T et al (2020) Estimation of the asymptomatic ratio of novel coronavirus infections (COVID-19). 
Int J Infect Dis 94:154-155. https://doi.org/10.1016/j.ijid.2020.03. 020

Oran DP, Topol EJ (2020) Prevalence of asymptomatic SARS-CoV-2 infection: a narrative review. Ann Intern Med. https://doi.org/10. 7326/M20-3012

Poyiadji N, Shahin G, Noujaim D et al (2020) Covid-19-associated acute hemorrhagic necrotizing encephalopathy: CT and MRI features. Radiology 296(2):E119-E120. https://doi.org/10.1148/radiol. 2020201187

Rodriguez-Morales AJ et al (2020) Clinical, laboratory and imaging features of COVID-19: a systematic review and meta-analysis. Travel Med Infect Dis. 34:101623. https://doi.org/10.1016/j.tmaid.2020. 101623

Salari N, Hosseinian-Far A, Jalali R et al (2020) Prevalence of stress, anxiety, depression among the general population during the COVID-19 pandemic: a systematic review and meta-analysis. Global Health 16(1):57. https://doi.org/10.1186/s12992-02000589-w

Singhal T (2020) A review of Coronavirus Disease-2019 (COVID-19). Indian J Pediatr. 87(4):281-286. https://doi.org/10.1007/s12098020-03263-6

Siordia JA Jr (2020) Epidemiology and clinical features of COVID-19: a review of current literature. J Clin Virol. 127:104357. https://doi. org/10.1016/j.jcv.2020.104357

Song Y, Liu P, Shi XL et al (2020) SARS-CoV-2 induced diarrhoea as onset symptom in patient with COVID-19. Gut. 69(6):1143-1144. https://doi.org/10.1136/gutjnl-2020-320,891

TSB reports: COVID-19 in Bangladesh (2020) General holidays likely to be further extended till May 16. https://tbsnews.net/coronaviruschronicle/covid-19-bangladesh/govt-extend-general-holidays-tillmay-16-76291

Vetter P et al (2020) Clinical features of covid-19. BMJ 369:m1470. https://doi.org/10.1136/bmj.m1470

Wang C, Pan R, Wan X et al (2020) Immediate psychological responses and associated factors during the initial stage of the 2019 Coronavirus Disease (COVID-19) epidemic among the general population in China. Int J Environ Res Public Health 17(5):1729. https://doi.org/10.3390/ijerph17051729

World Health Organization (2020a) Mental health and COVID-19. https://www.who.int/teams/mental-health-and-substance-use/covid19

World Health Organization (2020b) Assessment of risk factors for coronavirus disease 2019 (COVID-19) in health workers: protocol for a case-control study. https://www.who.int/publications/i/item/ assessment-of-risk-factors-for-coronavirus-disease-2019-(covid19)-in-health-workers-protocol-for-a-case-control-study

Worldometer reports (2020) Coronavirus (COVID-19) Mortality Rate. https://www.worldometers.info/coronavirus/coronavirus-death-rate/

Zhang JJ, Dong X, Cao YY et al (2020) Clinical characteristics of 140 patients infected with SARS-CoV-2 in Wuhan, China. Allergy 75(7):1730-1741. https://doi.org/10.1111/all.14238

Zhong BL, Luo W, Li HM et al (2020) Knowledge, attitudes, and practices towards COVID-19 among Chinese residents during the rapid rise period of the COVID-19 outbreak: a quick online crosssectional survey. Int J Biol Sci. 16(10):1745-1752. https://doi.org/ $10.7150 /$ ijbs. 45221

Zhou F, Yu T, Du R et al (2020) Clinical course and risk factors for mortality of adult in patients with COVID-19 in Wuhan, China: a retrospective cohort study. Lancet 395(10229):1054-1062. https:// doi.org/10.1016/S0140-6736(20)30566-3

Zhu N, Zhang D, Wang W et al (2020a) A novel coronavirus from patients with pneumonia in China, 2019. N Engl J Med. 382(8): 727-733. https://doi.org/10.1056/NEJMoa2001017

Zhu Z et al (2020b) COVID-19 in Wuhan immediate psychological impact on 5062 health workers. BioRxiv. https://doi.org/10.1101/ 2020.02.20.20025338

Zou L, Ruan F, Huang M et al (2020) SARS-CoV-2 viral load in upper respiratory specimens of infected patients. N Engl J Med. 382(12): 1177-1179. https://doi.org/10.1056/NEJMc2001737

Publisher's note Springer Nature remains neutral with regard to jurisdictional claims in published maps and institutional affiliations. 